\title{
PERSPECTIVE \\ Ten Ways Artificial Intelligence Will Transform Primary Care
}

\author{
Steven Y. Lin, $M D^{7}$, Megan R. Mahoney, $M D^{7}$, and Christine A. Sinsky, $M D^{2}$ \\ 'Division of Primary Care and Population Health, Department of Medicine, Stanford University School of Medicine, Stanford, CA, USA; ${ }^{2}$, American \\ Medical Association, Chicago, IL, USA.
}

Artificial intelligence (AI) is poised as a transformational force in healthcare. This paper presents a current environmental scan, through the eyes of primary care physicians, of the top ten ways AI will impact primary care and its key stakeholders. We discuss ten distinct problem spaces and the most promising AI innovations in each, estimating potential market sizes and the Quadruple Aims that are most likely to be affected. Primary care is where the power, opportunity, and future of AI are most likely to be realized in the broadest and most ambitious scale. We propose how these AI-powered innovations must augment, not subvert, the patient-physician relationship for physicians and patients to accept them. AI implemented poorly risks pushing humanity to the margins; done wisely, AI can free up physicians' cognitive and emotional space for patients, and shift the focus away from transactional tasks to personalized care. The challenge will be for humans to have the wisdom and willingness to discern AI's optimal role in twenty-first century healthcare, and to determine when it strengthens and when it undermines human healing. Ongoing research will determine the impact of AI technologies in achieving better care, better health, lower costs, and improved wellbeing of the workforce.

KEY WORDS: artificial intelligence; primary care; Quadruple Aim; patientphysician relationship.

J Gen Intern Med 34(8):1626-30

DOI: $10.1007 / \mathrm{s} 11606-019-05035-1$

(c) Society of General Internal Medicine 2019

\section{INTRODUCTION}

Artificial intelligence (AI) has been called the "stethoscope of the 21 st century". "With all of the allure, hype, fear, and promise of $\mathrm{AI}$ as a transformational force in healthcare, we explore the top ten ways AI will impact primary care and the Quadruple $\mathrm{Aim}^{2}$ for key stakeholders: primary care physicians, patients, health systems, and payers. In this Perspective, we will examine ten distinct problem spaces and the most promising $\mathrm{AI}$ innovations in each, estimating potential market sizes and the Quadruple Aims that are most likely to be affected (Fig. 1).

\section{Prior presentations None.}

Received January 29, 2019

Revised March 12, 2019

Accepted April 5, 2019

Published online May 14, 2019
For this discussion, we define AI broadly as any technology designed to mimic human cognitive functions-including techniques such as classic machine learning, modern deep learning, natural language processing, and robotics. ${ }^{3}$ Although the topics presented here may also apply equally to specialty care, we have decided to shine a spotlight on primary care. With over 500 million visits per year in the USA $-51 \%$ of all physician office visits, more than any other medical specialty ${ }^{4}$ - primary care is where the power, opportunity, and future of AI are most likely to be realized in the broadest and most ambitious scale. Finally, we propose how these AIpowered innovations must augment, not subvert, the patientphysician relationship in order for primary care physicians and patients to embrace them.

\section{RISK PREDICTION AND INTERVENTION}

In the USA, hospital costs for potentially preventable conditions account for $\$ 1$ in every $\$ 10$ of total hospital expenditures. ${ }^{5,6}$ This means that millions of hospital stays and up to $\$ 100$ billion a year ${ }^{7}$ might be prevented with better risk prediction and interventions in the ambulatory setting. AIdriven predictive modeling with EHR data can now outperform traditional predictive models in forecasting in-hospital mortality, 30-day unplanned readmission, prolonged length of stay, and all of a patient's final discharge diagnoses. ${ }^{8}$ Kaiser Permanente has partnered with KenSci to risk stratify congestive heart failure patients using AI. ${ }^{9}$ Ochsner Health System and Epic have created an AI using over one billion clinical data points to predict patient deterioration with $98 \%$ accuracy. ${ }^{9}$ This technology is now moving into ambulatory care: Banner Health is using BaseHealth's AI to predict risk on 100,000 members for 42 health conditions in order to lower preventable ED visits and hospitalizations through primary care interventions. ${ }^{10}$

\section{POPULATION HEALTH MANAGEMENT}

As the USA moves away from fee-for-service to value-based payments, the size of the population health management industry is expected to reach $\$ 89$ billion by $2025 .{ }^{11}$ Key market players such as Allscripts, athenahealth, Cerner, eClinicalWorks, Enli, Epic, Evolent, Optum, and Philips are exploring the role of AI to improve their platforms' ability to 


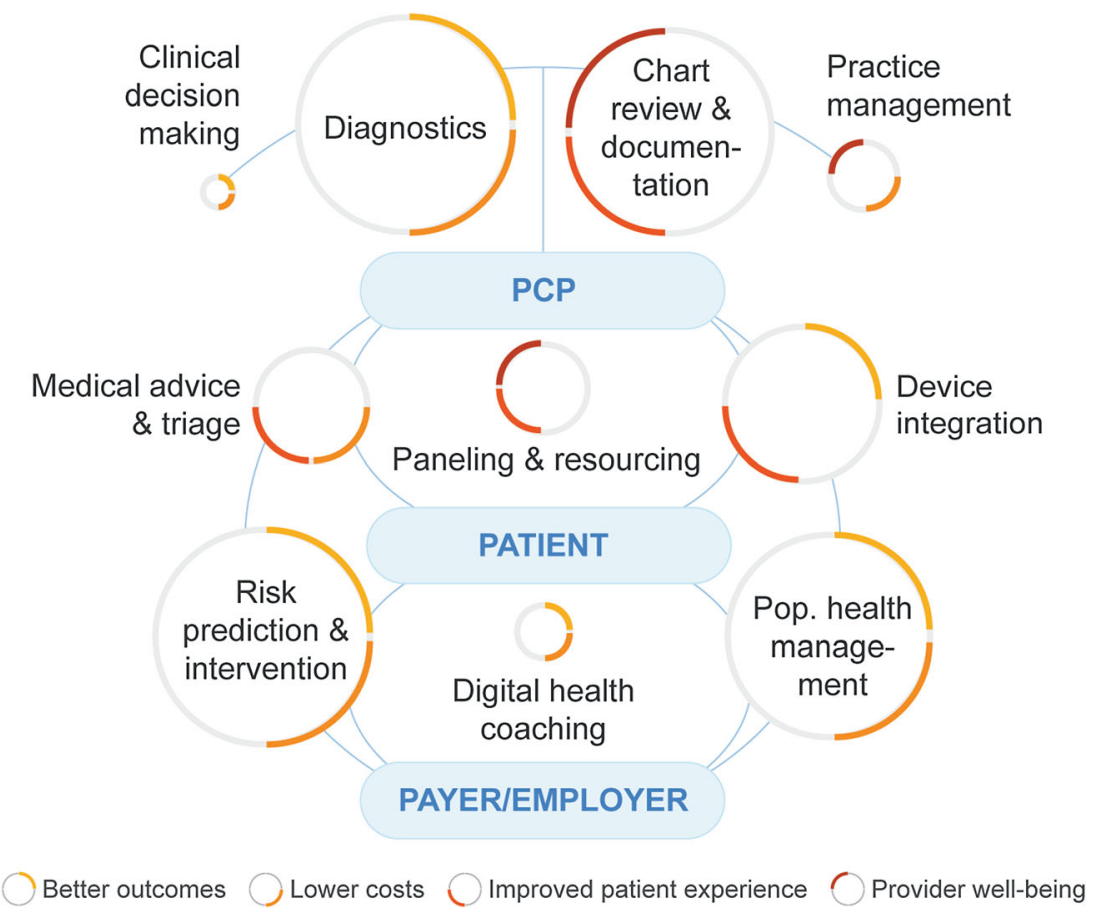

Figure 1 Top 10 ways artificial intelligence (AI) will impact primary care. Each problem space is represented by a circle; colored quadrants indicate the Quadruple Aims that are most impacted. Size of the circles is the estimate of the market value of each problem space in billions of dollars (USD)/year in the USA: risk prediction and intervention, \$100B; population health management, \$89B; medical advice/triage; \$27B; risk-adjusted paneling/resourcing, \$17B; device integration, \$52B; digital health coaching, \$6B; chart review and documentation, \$90B; diagnostics, \$100B; clinical decision making, \$1-2B; practice management, $\$ 10 \mathrm{~B}$. $\mathrm{PCP}=$ primary care physician.

identify and close care gaps, and to optimize their performance with the quality payment programs of MACRA. ${ }^{12}$ IBM and Siemens, for example, teamed up to bring Watson's AI into a new generation of population health tools for primary care physicians. $^{13}$

\section{MEDICAL ADVICE AND TRIAGE}

Many companies such as Babylon Health, Health Tap, Ada, Buoy, and Your.MD have developed "AI doctors"14 that provide health advice directly to patients with common symptoms, freeing up primary care access for more complex care. Babylon reported diagnostic accuracy comparable to human doctors. ${ }^{15}$ By 2025, the market for these services (using the current telemedicine market ${ }^{16}$ and retail clinics market ${ }^{17}$ as comparison) is projected to be $\$ 27$ billion a year. Insurer Prudential Asia recently negotiated a $\$ 100$ million licensing deal with Babylon Health to exclusively use its AI-powered software for its own apps across 12 Asian countries. ${ }^{18}$ Rather than AI replacing real providers for some conditions, we believe that AI support can be integrated into team-based care models that make it easier for primary care physicians to manage a patient panel. ${ }^{19}$

\section{RISK-ADJUSTED PANELING AND RESOURCING}

Risk-adjusted paneling can ensure that primary care physicians have adequate time to address the needs of each patient by increasing or decreasing panel sizes based on patient complexity, which can contribute to patient satisfaction and better work-life balance for physicians. Burnout-related turnover may cost US health systems tens of billions per year. ${ }^{20}$ The lack of established models for risk-adjusting panel size has led to potential AI solutions. At UCSF, EHR data on healthcare utilization was applied to train algorithms that were used to weigh panel sizes in primary care. ${ }^{21}$ In the future, such models can be used to determine the level of staffing support needed for primary care practices (e.g., medical assistants, nurses, advanced practice providers, clinical pharmacists, social workers) based on the complexity and intensity of care provided.

\section{DEVICE INTEGRATION}

Nearly one in four Americans own a wearable device that tracks vital signs or other health measures. ${ }^{22}$ The wearable device market is expected to reach $\$ 52$ billion by $2022 .^{23}$ Primary care physicians may be able to use data from such devices to diagnose or treat disease at earlier stages; however, the data's sheer volume and incompatibility with current EHRs make this unfeasible without the help of AI. Apple's HealthKit is an example of a tool that integrates data from multiple wearable devices into the EHR, enabling teams to track trends and spot any deviations that suggest illness. 


\section{DIGITAL HEALTH COACHING}

Management of chronic disease accounts for the majority of healthcare cost in the USA; 1 in 7 healthcare dollars are spent caring for people with diabetes alone, with a total estimated cost for diabetes over $\$ 300$ billion a year. ${ }^{24}$ Companies like Lark, Omada, Livongo, Glooko, and Virta are offering digital health coaching for diabetes, hypertension, and obesity. Similar programs that are integrated in health systems (such as Iora, Humana, and Caremore) have shown reductions in cost per patient through reduced office and hospital visits. ${ }^{25}$ Pure AI-driven fully automated text-based health coaching (provided by companies like Lark and Nutrino) has been shown to achieve weight loss comparable to in-person lifestyle interventions. ${ }^{26}$

\section{CHART REVIEW AND DOCUMENTATION}

Clinical documentation in the EHR is one of the biggest drivers of physician burnout in the USA, causing as much as $\$ 90-140$ billion in lost physician time per year. ${ }^{27}$ Technology companies with expertise in automatic speech recognition are teaming up with health systems - including Google and Stanford, ${ }^{28}$ Microsoft and UPMC, ${ }^{29}$ Nuance and Epic, ${ }^{30}$ and NoteSwift and athenahealth, ${ }^{31}$ plus a handful of startups such as Saykara and Suki - to develop AI-driven digital scribes that can listen in on patient-physician conversations and automatically generate a clinical note. Although still in its infancy, this type of AI carries the potential to unshackle physicians from the EHR and reduce burnout. ${ }^{27}$

\section{DIAGNOSTICS}

AI-powered algorithms for diagnosing disease is now outperforming physicians in detecting skin cancer, ${ }^{32}$ breast cancer, ${ }^{33}$ colorectal cancer, ${ }^{34}$ brain cancer, ${ }^{35}$ and cardiac arrhythmias. ${ }^{36}$ In regions with lack of access to specialty care, these tools in the hands of primary care doctors can provide significant benefit to patients. University of Iowa Health Care is using IDx-DR, an AI capable of detecting diabetic retinopathy to improve care. ${ }^{37}$ Aysa, an app from VisualDx, allows patients to take photos of their skin conditions: the AI will generate possible diagnoses and suggest self-care versus a visit to their physician. ${ }^{38}$ Google can accurately predict cardiovascular risk using retinal scans, ${ }^{39}$ and Tencent's AI can spot Parkinson's using smartphone videos ${ }^{40}$ — promising tools that can expand access to care and empower primary care physicians to broaden the services they can provide to patients. This could reduce the need for unnecessary referrals, increase continuity with patients, and enhance mastery for primary care physicians.

\section{CLINICAL DECISION MAKING}

Leading EHR suppliers-including Allscripts, athenahealth, Cerner, eClinicalWorks, and Epic - have all announced plans to add AI into their EHR workflows to support doctors in their clinical decision making. ${ }^{41}$ The next generation of clinical decision making platforms must move beyond alerts and best practice advisories to provide analytics and evidence-based content in the clinical workflow to drive intended actions. ${ }^{42}$ eClinicalWorks, for example, is developing a new version of their EHR that will feature an AI assistant that gives users evidence-based clinical suggestions in real time. ${ }^{43}$

\section{PRACTICE MANAGEMENT}

One of the most anticipated applications of AI in medicine is the automation of repetitive clerical tasks that are suffocating practices. For example, Olive uses AI to automate things like eligibility checks, insurance claims, prior authorizations, appointment reminders, billing, data reporting, and analytics. Other companies, such as Apixio and $3 \mathrm{M}$, have developed AI-powered risk adjustment and hierarchical condition category (HCC) auditors to aid practices in optimizing their coding for quality payment programs. ${ }^{44} \mathrm{AI}$ can also be used to automate certain aspects of pre-visit planning to make primary care encounters more efficient and rewarding for patients and physicians. ${ }^{45}$

\section{AUGMENTING (NOT SUBVERTING) THE PATIENT- PHYSICIAN RELATIONSHIP}

Two decades ago, the promises of EHRs were widely anticipated - no more guessing about your patients' medications, hunting for test results, or managing patients blindly without their records. Some of these promises came to pass, but many initial expectations were not met while new pain points emerged, most notably a fracture in the patientphysician relationship. EHRs were quickly installed without strong evidence to guide their design, implementation, and regulation, and have contributed to a highly transactional model, with care signified by tick boxes, communication by smartphrases, and where screen-time has replaced face-time as the primary act of healthcare. ${ }^{46,47}$ It is no wonder widespread burnout among physicians has resulted. ${ }^{48,49}$

For AI to add the most value and for physicians to embrace it, these innovations should support, not supplant, the patientphysician relationship. Healthcare is fundamentally a social enterprise, powered by committed, caring, and collaborative connections between the humans involved. AI implemented poorly risks pushing humanity to the margins; implemented wisely, AI can free up physicians' cognitive and emotional space for their patients, even helping them to become better at being human. ${ }^{50}$ 
As one example, AI health coaches connected to a real person in the care team could provide the frequent touches a patient needs to follow through on lifestyle interventions. On the other hand, a one-size-fits-all coach that resides completely detached from the care team may feel alienating to the patient. There is emerging evidence that AI, when implemented as part of a human care team, is acceptable to both patients and providers. A text-based conversational AI for weight loss had a patient trust and satisfaction score of $87 \%^{26}$; an AIdriven diabetic retinopathy screening protocol providing realtime results generated a $96 \%$ satisfaction score with $78 \%$ of patients preferring the AI model over usual care ${ }^{51}$; humanoid robot assistants for medication management have shown a high degree of acceptability (86-100\%) with patients across the age spectrum, from children with type 1 diabetes ${ }^{52}$ to elderly home-care patients. ${ }^{53}$ More research into AI applications, with studies investigating patient engagement and acceptability, and how best to integrate AI into team-based care models, should be pursued.

We believe that the optimal role of AI is to free up physicians' cognitive and emotional space for their patients. Using the "cockpit" analogy, ${ }^{54}$ AI has the power to protect the pilot (the physician) from the unsafe distractions of information overload by organizing the cacophony of patient data, evidence-based practice guidelines, and compliance monitoring check boxes, into a "manageable cockpit" for primary care physicians. It can also automate repetitive and cumbersome tasks, such as documentation and other administrative distractions, that contribute to a hazardous cockpit for physicians. Undivided attention with compassion is the most powerful diagnostic and therapeutic tool physicians can provide their patients. AI will be most effective when it enhances physicians' ability to focus their full attention on the patient by shifting the physicians' responsibilities away from transactional tasks toward personalized care ${ }^{55}$ that lies at the heart of human healing.

As AI becomes the second great wave of technological innovations to offer power and possibility for modern healthcare, a key question is: will AI augment, rather than subvert, relationships? Or will managing and being managed by $\mathrm{AI}$ add yet another technological master and burden to the lives of physicians? The human challenge will be to have the wisdom and willingness to discern AI's optimal role, and to determine when it strengthens and when it undermines human healing. Ongoing research will be needed to determine the impact of AI in achieving the Quadruple Aim of better care, better health, lower costs, and improved well-being of the workforce.

Acknowledgments: The authors thank Rebecca Rolfe, MS, for her creative and technical support on the manuscript figure.

Corresponding Author: Steven Y. Lin, MD; Division of Primary Care and Population Health, Department of Medicine, Stanford University School of Medicine, Stanford, CA, USA (e-mail: stevenlin@stanford. $e d u$.

\section{Compliance with Ethical Standards:}

Conflict of Interest: SYL is the PI on a research project sponsored by Google to understand how deep learning techniques and automatic speech recognition can facilitate clinical documentation. Google had no role in the preparation of this manuscript and the decision to approve publication of the finished manuscript. SYL has no financial interests to declare.

MRM and SYL collaborated with Babylon Health to write clinical case vignettes to train a triage and diagnosis software. Babylon Health had no role in the preparation of this manuscript and the decision to approve publication of the finished manuscript. Neither have any conflicts of interest to declare.

CAS has no conflicts of interest to declare.

\section{REFERENCES}

1. Mesko B. Artificial Intelligence is the Stethoscope of the 21st Century. The Medical Futurist. https://medicalfuturist.com/ibm-watson-is-thestethoscope-of-the-21st-century. Updated July 18, 2017. Accessed 7 March 2019.

2. Bodenheimer T, Sinsky C. From triple to quadruple aim: care of the patient requires care of the provider. Ann Fam Med. 2014;12(6):573-576.

3. Esteva A, Robicquet A, Ramsundar B, et al. A guide to deep learning in healthcare. Nat Med. 2019;25(1):24-29.

4. Rui P, Okeyode T. National Ambulatory Medical Care Survey: 2015 State and National Summary Tables. National Center for Health Statistics, Centers for Disease Control and Prevention. https://www.cdc.gov/nchs/ data/ahcd/namcs_summary/2015_namcs_web_tables.pdf. Accessed 7 March 2019.

5. Jiang HJ, Russo CA, Barrett ML. Nationwide Frequency and Costs of Potentially Preventable Hospitalizations, 2006. HCUP Statistical Brief \#72. U.S. Agency for Healthcare Research and Quality, Rockville, MD. https://www.hcup-us.ahrq.gov/reports/statbriefs/sb72.pdf. Updated April 2009. Accessed 7 March 2019.

6. Fingar KR, Barrett ML, Elixhauser A, Stocks C, Steiner CA. Trends in Potentially Preventable Inpatient Hospital Admissions and Emergency Department Visits. HCUP Statistical Brief \#195. U.S. Agency for Healthcare Research and Quality, Rockville, MD. https://www.hcup-us. ahrq.gov/reports/statbriefs/sb195-Potentially-Preventable-Hospitalizations.pdf. Updated November 2015. Accessed 7 March 2019.

7. American Hospital Association. Fast Facts on U.S. Hospitals, 2018. https://www.aha.org/statistics/fast-facts-us-hospitals. Updated February 2018. Accessed 7 March 2019.

8. Rajkomar A, Oren E, Chen $\mathbf{K}$, et al. Scalable and accurate deep learning with electronic health records. Nat Digital Med. 2018;1:18.

9. Microsoft Industry Blogs. Winners of the 2018 Microsoft Health Innovation Awards. https://cloudblogs.microsoft.com/industry-blog/industry/ health/winners-of-the-2018-microsoft-health-innovation-awards/. Updated March 7, 2018. Accessed 7 March 2019.

10. BaseHealth. Predictive Health Platform Optimizes Health and Disease Risk Management, Improves Population Health Outcomes. https://www. basehealth.com/news/2018/02/27/predictive-health-platform-optimizes-health-and-disease-risk-management-improves-populationhealth-outcomes.html. Updated February 27, 2018. Accessed 7 March 2019.

11. Grand View Research. Population Health Management Market Size Worth $\$ 88.9$ Billion By 2025. https://www.grandviewresearch.com/press-release/global-population-health-management-phm-market. Updated April 2018. Accessed 7 March 2019.

12. Centers for Medicare \& Medicaid Services. Value-Based Programs. https://www.cms.gov/Medicare/Quality-Initiatives-Patient-AssessmentInstruments/Value-Based-Programs/Value-Based-Programs.html. Updated July 25, 2018. Accessed 7 March 2019.

13. Japsen B. IBM Watson, Siemens Partner To Tap Population Health Industry. Forbes. https://www.forbes.com/sites/brucejapsen/2016/10/ 11/ibm-watson-siemens-partner-to-tap-population-health-industry/ \#438447e66600. Updated October 11, 2016. Accessed 7 March 2019.

14. Mukherjee S. You Can Now Download an Artificial Intelligence Doctor. Fortune. http://fortune.com/2017/01/10/healthtap-dr-ai-launch/. Updated January 10, 2017. Accessed 7 March 2019.

15. Razzaki S, Baker A, Perov Y, et al. A comparative study of artificial intelligence and human doctors for the purpose of triage and diagnosis. Babylon Health. https://www.researchgate.net/publication/ 326056790_A_comparative_study_of_artificial_intelligence_and_human_ 
doctors_for_the_purpose_of_triage_and_diagnosis. Updated June 2018. Accessed 7 March 2019

16. Landi H. Telehealth Market Estimated to Reach $\$ 19.5 B$ by 2025 . Healthcare Innovation. https://www.healthcare-informatics.com/newsitem/telemedicine/report-telehealth-market-estimated-reach-195b2025. Updated April 2, 2018. Accessed 7 March 2019.

17. Grand View Research. U.S. Retail Clinics Market Worth $\$ 7.3$ Billion by 2025. https://www.grandviewresearch.com/press-release/us-retailclinics-market-analysis. Updated September 2017. Accessed 7 March 2019.

18. Olson P. Rise Of The AI-Doc: Insurer Prudential Taps Babylon Health In \$100 Million Software Licensing Deal. Forbes. https://www.forbes.com/ sites/parmyolson/2018/08/02/rise-of-the-ai-doc-insurer-prudentialtaps-babylon-health-in-100-million-software-licensing-deal/ \#386d868d628f. Updated August 2, 2018. Accessed 7 March 2019.

19. Smith CD, Balatbat C, Corbridge S, et al. Implementing Optimal TeamBased Care to Reduce Clinician Burnout. NAM Perspectives. Discussion Paper, National Academy of Medicine, Washington, DC. https://nam. edu/implementing-optimal-team-based-care-to-reduce-clinician-burnout/. Updated September 17, 2018. Accessed 7 March 2019.

20. Shanafelt T, Goh J, Sinsky C. The Business Case for Investing in Physician Well-being. JAMA Intern Med. 2017;177(12):1826-1832.

21. Rajkomar A, Yim JW, Grumbach K, Parekh A. Weighting Primary Care Patient Panel Size: A Novel Electronic Health Record-Derived Measure Using Machine Learning. JMIR Med Inform. 2016;4(4):e29.

22. Adams A, Shankar M, Tecco H. 50 Things We Now Know About Digital Health Consumers. Rock Health. https://rockhealth.com/reports/digital-health-consumer-adoption-2016/. Updated 2016. Accessed 7 March 2019.

23. Market Research Engine. Wearable Devices Market By Product Analysis, By Application Analysis, and By Regional Analysis - Global Forecast by 2016-2022. https://www.marketresearchengine.com/wearable-devicesmarket. Updated July 2017. Accessed 7 March 2019.

24. American Diabetes Association. The Cost of Diabetes. http://www. diabetes.org/advocacy/news-events/cost-of-diabetes.html. Updated March 22, 2018. Accessed 7 March 2019.

25. Jahns RG. Research: 6 Success Factors for Digital-Enabled Health Coaching. HIT Consultant. https://hitconsultant.net/2018/02/09/success-factors-for-digital-enabled-health-coaching/. Updated February 9, 2018. Accessed 7 March 2019.

26. Stein N, Brooks K. A Fully Automated Conversational Artificial Intelligence for Weight Loss: Longitudinal Observational Study Among Overweight and Obese Adults. JMIR Diabetes. 2017;2(2):e28.

27. Lin SY, Shanafelt TD, Asch SM. Reimagining Clinical Documentation With Artificial Intelligence. Mayo Clin Proc. 2018;93(5):563-565.

28. Chou K, Chiu CC. Understanding Medical Conversations. Google AI Blog. https://ai.googleblog.com/2017/11/understanding-medical-conversations.html. Updated November 21, 2017. Accessed 7 March 2019.

29. McGrane C. Microsoft and UPMC unveil virtual AI assistant that listens in and takes notes on doctor's visits. GeekWire. https://www.geekwire. com/2018/microsoft-healthcare/. Updated February 28, 2018. Accessed 7 March 2019.

30. Spitzer J. Nuance brings 1st integrated virtual assistant to Epic EHR. Becker's Hospital Review. https://www.beckershospitalreview.com/ehrs/ nuance-brings-1st-integrated-virtual-assistant-to-epic-ehr.html. Updated September 7, 2018. Accessed 7 March 2019.

31. Miliard M. Athenahealth partners with NoteSwift on AI-powered EHR documentation. Healthcare IT News. https://www.healthcareitnews. $\mathrm{com} /$ news/athenahealth-partners-noteswift-ai-powered-ehr-documentation. Updated January 31, 2018. Accessed 7 March 2019.

32. Haenssle HA, Fink C, Schneiderbauer R, et al. Man against machine: diagnostic performance of a deep learning convolutional neural network for dermoscopic melanoma recognition in comparison to 58 dermatologists. Ann Oncol. 2018;29(8):1836-1842.

33. Liu Y, Kohlberger T, Norouzi M, et al. Artificial Intelligence-Based Breast Cancer Nodal Metastasis Detection [published online ahead of print: October 8, 2018]. Arch Pathol Lab Med. doi:https://doi.org/10.5858/ arpa.2018-0147-OA.

34. Mukherjee S. This New AI Can Detect a Deadly Cancer Early With $86 \%$ Accuracy. Fortune. http://fortune.com/2017/10/30/ai-early-cancer-detection/. Updated October 30, 2017. Accessed 7 March 2019.

35. Chinese YL, Beats AI. Doctors in Diagnosing Brain Tumors. Popular Mechanics. https://www.popularmechanics.com/technology/robots/ a22148464/chinese-ai-diagnosed-brain-tumors-more-accurately-physicians/. Updated July 14, 2018. Accessed 7 March 2019.

36. Hannun AY, Rajpurkar P, Haghpanahi M, et al. Cardiologist-level arrhythmia detection and classification in ambulatory electrocardiograms using a deep neural network. Nat Med. 2019;25(1):65-69.

37. IDx. University of Iowa Health Care First to Adopt IDx-DR in a Diabetes Care Setting. Cision PR Newswire. https://www.prnewswire.com/newsreleases/university-of-iowa-health-care-first-to-adopt-idx-dr-in-a-diabetes-care-setting-300672070.html. Updated June 26, 2018. Accessed 7 March 2019.

38. VisualDx. VisualDx to Launch AI-Enabled Smart Symptom Checker. Cision PR Newswire. https://www.prnewswire.com/news-releases/ visualdx-to-launch-ai-enabled-smart-symptom-checker-300697148. html. Updated August 15, 2018. Accessed 7 March 2019

39. Poplin R, Varadarajan AV, Blumer $\mathbf{K}$, et al. Prediction of cardiovascular risk factors from retinal fundus photographs via deep learning. Nat Biomed Eng. 2018;2:158-164.

40. Shead S. Tencent Aims to Train AI To Spot Parkinson's In 3 Minutes. Forbes. https://www.forbes.com/sites/samshead/2018/10/08/ tencent-aims-to-train-ai-to-spot-parkinsons-in-3-minutes / \#52451b5b6f36. Updated October 8, 2018. Accessed 7 March 2019.

41. Sullivan T. Next up for EHRs: Vendors adding artificial intelligence into the workflow. Healthcare IT News. https://www.healthcareitnews.com/ news/next-ehrs-vendors-adding-artificial-intelligence-workflow. Updated March 13, 2018. Accessed 7 March 2019.

42. Shortliffe EH, Sepulveda MJ. Clinical Decision Support in the Era of Artificial Intelligence. JAMA. 2018;320(21):2199-2200.

43. Sullivan T. eClinicalWorks CEO Girish Navani: Our next EHR will be like a Bloomberg Terminal. Healthcare IT News. https://www. healthcareitnews.com/news/eclinicalworks-ceo-girish-navani-our-nextehr-will-be-bloomberg-terminal. Updated March 7, 2018. Accessed 7 March 2019.

44. Apixio. Apixio Launches HCC Auditor, the First AI-Powered Risk Adjustment Auditing Solution. https://globenewswire.com/news-release/2018/07/25/1541847/0/en/Apixio-Launches-HCC-Auditor-theFirst-AI-Powered-Risk-Adjustment-Auditing-Solution.html. Updated July 25, 2018. Accessed 7 March 2019.

45. Sinsky CA, Sinsky TA, Rajcevich E. Putting Pre-Visit Planning Into Practice. Fam Pract Manag. 2015;22(6):30-38.

46. Arndt BG, Beasley JW, Watkinson MD, et al. Tethered to the EHR: Primary Care Physician Workload Assessment Using EHR Event Log Data and Time-Motion Observations. Ann Fam Med. 2017;15(5):419-426.

47. Sinsky C, Colligan $\mathbf{L}, \mathbf{L i} \mathbf{L}$, et al. Allocation of Physician Time in Ambulatory Practice: A Time and Motion Study in 4 Specialties. Ann Intern Med. 2016;165(11):753-760.

48. Shanafelt TD, Dyrbye LN, Sinsky C, et al. Relationship Between Clerical Burden and Characteristics of the Electronic Environment With Physician Burnout and Professional Satisfaction. Mayo Clin Proc. 2016;91(7):836-848.

49. Shanafelt TD, Hasan O, Dyrbye LN. Changes in Burnout and Satisfaction With Work-Life Balance in Physicians and the General US Working Population Between 2011 and 2014. Mayo Clin Proc. 2015;90(12):16001613.

50. Israni ST, Verghese A. Humanizing Artificial Intelligence. JAMA. 2019;321(1):29-30.

51. Keel S, Lee PY, Scheetz J, et al. Feasibility and patient acceptability of a novel artificial intelligence-based screening model for diabetic retinopathy at endocrinology outpatient services: a pilot study. Sci Rep. 2018;8(1):4330.

52. Al-Taee MA, Kapoor R, Garrett C, Choudhary P. Acceptability of Robot Assistant in Management of Type 1 Diabetes in Children. Diabetes Technol Ther. 2016;18(9):551-554.

53. Rantanen P, Parkkari T, Leikola S, Airaksinen M, Lyles A. An In-home Advanced Robotic System to Manage Elderly Home-care Patients' Medications: A Pilot Safety and Usability Study. Clin Ther. 2017;39(5):1054-1061.

54. Sinsky CA, Privitera MR. Creating a "Manageable Cockpit" for Clinicians: A Shared Responsibility. JAMA Intern Med. 2018;178(6):741-742.

55. Reuben DB, Sinsky CA. From Transactional Tasks to Personalized Care: A New Vision of Physicians' Roles. Ann Fam Med. 2018;16(2):168-169.

Publisher's Note Springer Nature remains neutral with regard to jurisdictional claims in published maps and institutional affiliations. 